\title{
Some common fixed point theorems for $(\Phi, \Psi)$-weak contractions in intuitionistic generalized fuzzy cone metric spaces
}

\author{
M. Jeyaraman ${ }^{1}$ and S. Sowndrarajan ${ }^{2 *}$
}

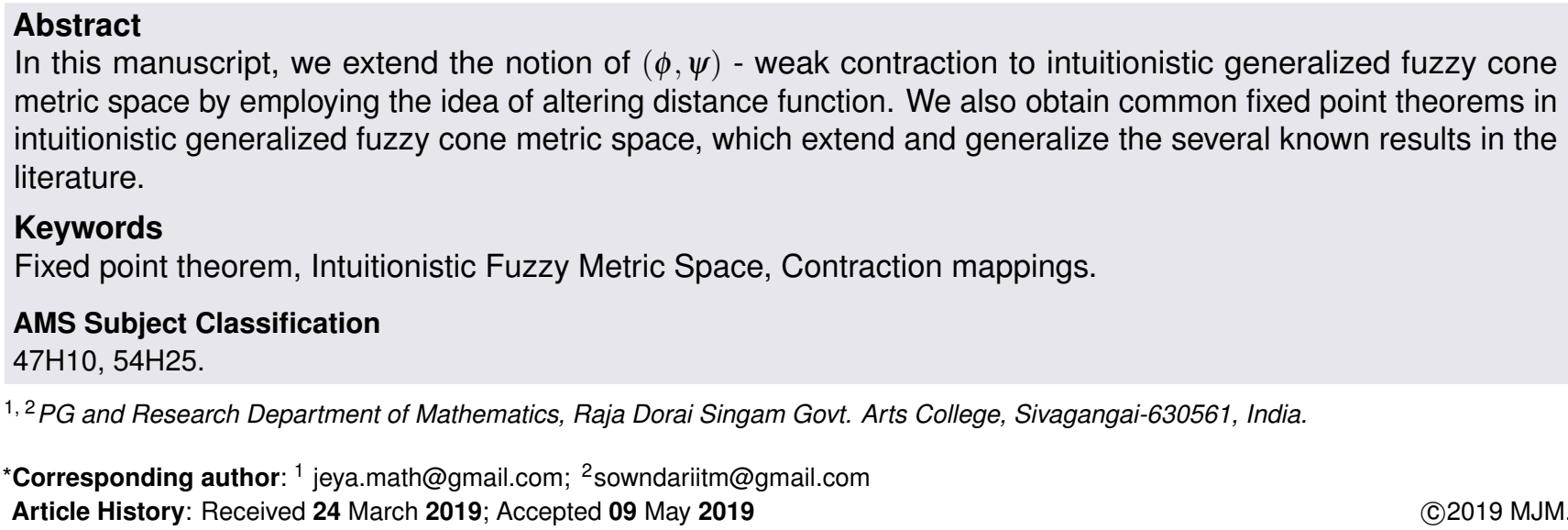

\section{Contents}

1 Introduction ........................... 154

2 Preliminaries ............................... 154

3 Main Results ......................... 156

4 Conclusion ............................ 158

References ........................... 158

\section{Introduction}

In 1965 Zadeh [16] defined fuzzy set which is a real tool for the understanding of many phenomena, not only in the field of mathematics but also in other fields of science. Atanassov [3] bring a new notion called intuitionistic fuzzy set which provides a very suitable tool to describe the uncertain or impressive decision information. In 2007 Shaban Sedghi [14] introduced $D^{*}$-metric which is a probable modification of the definition of D-metric introduced by Dhage. Park [11] gave the notion of intuitionistic fuzzy metric space which is a generalization of fuzzy metric space due to George and Veeramani [4]. Huang and Zhang [5] defined the concept of cone metric space and proved some fixed point theorems for contractive mappings. Later Tarken Oner et al [9] introduced fuzzy cone metric space that generalized the corresponding notions of fuzzy metric space. In 1984, Khan et al [8] employed the idea of altering distane function in metric fixed point results. In 2010 Vetro et al [15] defined the notion of $(\phi, \psi)$ - weak contraction in fuzzy metric space. Recently, Bag et al [2] extend the notion of $(\phi, \psi)$-weak contraction in intuitionistic fuzzy metric space and proved some fixed point theorems by using the altering distance function. For more results on fuzzy metric space and fuzzy cone metric space one can see the research papers in ([1], [6], [10], [12], [13]) The purpose of this paper is to extend and generalized the $(\phi, \psi)$-weak contraction to intuitionistic generalized fuzzy cone metric space and prove some common fixed point results.

\section{Preliminaries}

In this section, we recall some definitions and basic results of intuitionistic fuzzy cone metric space which will be used throughout the paper.

Definition 2.1. A binary operation $*:[0,1] \times[0,1] \rightarrow[0,1]$ is a continuous $t$-norm if $*$ satisfies the following conditions :

1. * is associative and commutative,

2. * is continuous, 
3. $a * 1=$ a for all $a \in[0,1]$,

4. $a * b \leq c * d$ whenever $a \leq c$ and $b \leq d$ for all $a, b, c$, $d \in[0,1]$.

Example 2.2. $a * b=\min \{a, b\}$ and $a * b=a b$

Definition 2.3. A binary operation $\diamond:[0,1] \times[0,1] \rightarrow[0,1]$ is said to be a continuous $t$-co norm if it satisfies the following conditions :

1. $\diamond$ is associative and commutative,

2. $\diamond$ is continuous,

3. $a \diamond 0=$ a for all $a \in[0,1]$,

4. $a \diamond b \leq c \diamond d$ whenever $a \leq c$ and $b \leq d$ for all $a, b, c$, $d \in[0,1]$.

Example 2.4. $a \diamond b=\max \{a, b\}$ and $a \diamond b=\min \{a+b, l\}$

Definition 2.5. Let $E$ be a real Banach space, $\theta$ be the zero of $E$ and $P$ a subset of $E$. Then $P$ is called a cone if and only if

1. $P$ is closed, nonempty and $P \neq\{\theta\}$,

2. if $a, b \in R, a, b \geq 0$ and $x, y \in P$, then $a x+b y \in P$,

3. if both $x \in P$ and $-x \in P$, then $x=\theta$.

For a given cone $\mathrm{P}$, a partial ordering $\preceq$ on $\mathrm{E}$ with respect to $\mathrm{P}$ is defined by $\mathrm{x} \preceq \mathrm{y}$ if and only if $\mathrm{y}-\mathrm{x} \in \mathrm{P}$. The notation $\mathrm{x} \prec \mathrm{y}$ will stand for $\mathrm{x} \preceq \mathrm{y}$ and $\mathrm{x} \neq \mathrm{y}$, while $\mathrm{x} \ll \mathrm{y}$ will stand for $\mathrm{y}-\mathrm{x} \in$ int $(\mathrm{P})$. Throughout this paper, we assume that all cones have non empty interior.

A cone $\mathrm{P}$ is called normal if there exists a constant $K>0$ such that for all $t, s \in E, \theta \preceq t \preceq s$ implies $\|t\| \leq K\|s\|$ and the least positive number $\mathrm{K}$ satisfying this property is called normal constant of $\mathrm{P}$.

Definition 2.6. A 3-tuple $(X, \mathscr{M}, *)$ is called a Generalized Fuzzy Cone Metric Space (Shortly GFCMS) if $P$ is a cone of $E, X$ is an arbitrary set, $*$ is continuous $t$-norm and $\mathscr{M}$ is a fuzzy set on $X^{3} \times \operatorname{int}(P)$ satisfying the following conditions; For all $x, y, z \in X$ and $t, s \in \operatorname{int}(P)$

1. $\mathscr{M}(x, y, z, t)>0$ for all $x, y, z \in X$,

2. $\mathscr{M}(x, y, z, t)=1$ if and only if $x=y=z$,

3. $\mathscr{M}(x, y, z, t)=\mathscr{M}(p\{x, y, z\}, t)$, where $p$ is permutation,

4. $\mathscr{M}(x, y, a, t) * \mathscr{M}(a, z, z, s) \leq M(x, y, z, t+s)$,

5. $\mathscr{M}(x, y, z,):. \operatorname{int}(P) \rightarrow[0,1]$ is continuous.
Example 2.7. Let $E=\mathbb{R}^{+}$, Then $P=\left\{\left(k_{1}, k_{2}, k_{3}\right): k_{1}, k_{2}, k_{3} \geq\right.$ $0\} \subseteq E$ is a normal cone with normal constant $K=1$. Let $X$ $=R, a * b=a b$ and $M: X^{3} \times \operatorname{int}(P) \rightarrow[0,1]$ defined by

$$
\mathscr{M}(x, y, z, t)=\frac{1}{e^{\frac{|x-y|+|y-z|+|z-x|}{|t| \mid}}}
$$

for all $x, y, z \in X$ and $t \gg \theta$.

Definition 2.8. Let $(X, \mathscr{M}, *)$ is said to be a Generalized Fuzzy Cone Metric space, if $x \in X$ and $\left\{x_{n}\right\}$ be a sequence in $X$. Then

1. $\left\{x_{n}\right\}$ is said to converge to $x$ if for any $t \gg \theta$ and any $r$ $\in(0,1)$ there is $n_{0} \in \mathbb{N}$ such that $\mathscr{M}\left(x_{m}, x, x, t\right)>1-r$ for all $m, n \geq n_{0}$.

2. $\left\{x_{n}\right\}$ is said to be a Cauchy sequence if for any $0<$ $\varepsilon<1$ and for any $t \gg \theta$ there is $n_{0} \in \mathbb{N}$ such that $\mathscr{M}\left(x_{m}, x_{n}, x_{l}, t\right)>1-\varepsilon$ for all $m, n, l \geq n_{0}$.

3. $(X, \mathscr{M}, *)$ is called complete if every Cauchy sequence is convergent in $X$.

Definition 2.9. A 5-tuple $(X, \mathscr{M}, \mathscr{N}, *, \diamond)$ is an intuitionistic generalized fuzzy cone metric space if $X$ is an arbitrary set, $*$ is a continuous t-norm, $\diamond$ is a continuous $t$-conorm and $\mathscr{M}, \mathscr{N}$ are fuzzy sets in $X^{3} \times$ int $(P)$ satisfying the following conditions:

1. $\mathscr{M}(x, y, z, t)+\mathscr{N}(x, y, z, t) \leq 1$,

2. $\mathscr{M}(x, y, z, t)>0$

3. $\mathscr{M}(x, y, z, t)=1$ if and only if $x=y=z$,

4. $\mathscr{M}(x, y, z, t)=\mathscr{M}(p\{x, y, z\}, t)$, where $p$ is permutation,

5. $\mathscr{M}(x, y, a, t) * \mathscr{M}(a, z, z, s) \leq \mathscr{M}(x, y, z, t+s)$,

6. $\mathscr{M}(x, y, z,):. \operatorname{int}(P) \rightarrow[0,1]$ is continuous,

7. $\mathscr{N}(x, y, z, t)<0$,

8. $\mathscr{N}(x, y, z, t)=0$ if and only if $x=y=z$,

9. $\mathscr{N}(x, y, z, t)=\mathscr{N}(p\{x, y, z\}, t)$, where $p$ is permutation,

10. $\mathscr{N}(x, y, a, t) * \mathscr{N}(a, z, z, s) \geq \mathscr{N}(x, y, z, t+s)$,

11. $\mathscr{N}(x, y, z,):. \operatorname{int}(P) \rightarrow[0,1]$ is continuous,

The functions $\mathscr{M}(\mathrm{x}, \mathrm{y}, \mathrm{z}, \mathrm{t})$ and $\mathscr{N}(\mathrm{x}, \mathrm{y}, \mathrm{z}, \mathrm{t})$ denote the degree of nearness and the degree of non-nearness between $x$, $\mathrm{y}$ and $\mathrm{z}$ with respect to $\mathrm{t}$ respectively.

Definition 2.10. Let $(X, d)$ be a metric space and let $P=\mathbb{R}^{+}$. Denote $a * b=a b$ and $a \diamond b=\min \{1, a+b\}$

for all $a, b \in[0,1]$ and let $\mathscr{M}$ and $\mathscr{N}$ be fuzzy sets on $X^{3} \times$ $\operatorname{int}(P)$ defined as follows:

$$
\mathscr{M}(x, y, z, t)=\frac{k t^{n}}{k t^{n}+l D^{*}(x, y, z)}
$$


and

$$
\mathscr{N}(x, y, z, t)=\frac{D^{*}(x, y, z)}{m t^{n}+l D^{*}(x, y, z)}
$$

for all $k, l, m, n \in \operatorname{int}(P)$ and for each $t \gg \theta$. Then $(X, \mathscr{M}, \mathscr{N}, *, \diamond)$ is an intuitionistic generalized fuzzy cone metric space.

Definition 2.11. Let $(X, \mathscr{M}, \mathscr{N}, *, \diamond)$ be an intuitionistic generalized fuzzy cone metric space, $x \in X$ and $\left\{x_{n}\right\}$ be a sequence in $X$. Then

1. $\left\{x_{n}\right\}$ is said to converge to $x$ iffor each $t \gg \theta$, we have $\lim _{n \rightarrow \infty} \mathscr{M}\left(x_{m}, x_{n}, x, t\right)=1$ and $\lim _{n \rightarrow \infty} \mathscr{N}\left(x_{m}, x_{n}, x, t\right)=$ 0 for all $m, n \geq n_{0}$.

2. $\left\{x_{n}\right\}$ is said to be a Cauchy sequence if for each $r \in$ $(0,1)$ and $t \gg \theta$, there exist $n_{0} \in \mathbb{N}$ such that $\mathscr{M}\left(x_{m}, x_{n}, x_{l}, t\right)>1-r$ and $\mathscr{N}\left(x_{m}, x_{n}, x_{l}, t\right)<r$ for all $m, n, l \geq n_{0}$.

3. $(X, \mathscr{M}, \mathscr{N}, *, \diamond)$ is called complete if every Cauchy sequence is convergence in $X$.

Definition 2.12. A function $\phi:[0, \infty) \rightarrow[0, \infty)$ is an altering distance function if $\phi(t)$ is monotone non-decreasing and continuous and $\phi(t)=0$ if and only if $t=\theta$.

Definition 2.13. Let $(X, \mathscr{M}, \mathscr{N}, *, \diamond)$ be an intuitionistic generalized fuzzy cone metric space and $f, g: X \rightarrow X$ be two mappings. The mapping $g$ is called intuitionistic $(\phi, \psi)-$ weak contraction with respect to $f$ if there exist a function $\psi:[0, \infty) \rightarrow[0, \infty)$ with $\psi(r)>0$ for $r>0$ and $\psi(r)=0$ and an alternating distance function $\phi$ such that

$$
\begin{aligned}
\phi\left(\frac{1}{\mathscr{M}(g(x), g(y), g(z), t)}-1\right) & \leq \phi\left(\frac{1}{\mathscr{M}(f(x), f(y), f(z), t)}-1\right) \\
& -\psi\left(\frac{1}{\mathscr{M}(f(x), f(y), f(z), t)}-1\right)
\end{aligned}
$$

$$
\begin{aligned}
\phi(\mathscr{N}(g(x), g(y), g(z), t) & \leq \phi(\mathscr{N}(f(x), f(y), f(z), t)) \\
& -\psi(\mathscr{N}(f(x), f(y), f(z), t))
\end{aligned}
$$

hold for all $x, y, z \in X$ and each $t \gg \theta$. If $f$ is the identity map, then $g$ is called intuitionistic $(\phi, \psi)$ - weak contraction mapping.

Definition 2.14. Let $(X, \mathscr{M}, \mathscr{N}, *, \diamond)$ be an intuitionistic generalized fuzzy cone metric space and $f, g: X \rightarrow X$ be two mappings. A point $u$ in $X$ is called coincidence point (common fixed point) of $f$ and $g$ if $z=f(u)=g(u)$.

Definition 2.15. Let $\left\{f_{i}\right\}$ and $\left\{g_{i}\right\}$ be two finite families of self mappings on $X$ are said to be pairwise commuting if
1. $f_{i} f_{j}=f_{j} f_{i}$, where $i, j \in\{1,2, \ldots, m\}$,

2. $g_{i} g_{j}=g_{j} g_{i}$, where $i, j \in\{1,2, \ldots, n\}$,

3. $f_{i} g_{j}=g_{j} f_{i}$, where $i \in\{1,2, \ldots, m\}$ and $j \in\{1,2, \ldots, n\}$.

\section{Main Results}

Theorem 3.1. Let $(X, \mathscr{M}, \mathscr{N}, *, \diamond)$ be an intuitionistic generalized fuzzy cone metric space and $g: X \rightarrow X$ be a intuitionistic generalized $(\phi, \psi)$ - weak contraction with respect to $f: X \rightarrow X$. If $g(X) \subseteq f(X)$ and $f(X)$ or $g(X)$ is a complete subset of $X$, then $f$ and $g$ have a unique common fixed point in $X$ provided that $\psi$ is a continuous function.

Proof. Let $x_{0} \in \mathrm{X}$ be an arbitrary point. Choose a point $x_{1} \in \mathrm{X}$ such that $g\left(x_{0}\right)=f\left(x_{1}\right)$. This can be done since $g(X) \subseteq f(X)$. Continuing this process, we obtain a sequence $\left\{x_{n}\right\}$ in $\mathrm{X}$ such that $y_{n}=g\left(x_{n}\right)=f\left(x_{n+1}\right)$.

We assume that $y_{n} \neq y_{n+1}$ for all $n \in \mathbb{N}$, otherwise $f$ and $g$ have a coincidence point. Now we get,

$$
\begin{aligned}
& \phi\left(\frac{1}{\mathscr{M}\left(y_{n}, y_{n}, y_{n+1}, t\right)}-1\right)=\phi\left(\frac{1}{\mathscr{M}\left(g\left(y_{n}\right), g\left(y_{n}\right), g\left(y_{n+1}\right), t\right)}-1\right) \\
& \leq \phi\left(\frac{1}{\mathscr{M}\left(f\left(x_{n}\right), f\left(x_{n}\right), f\left(x_{n+1}\right), t\right)}-1\right) \\
&-\psi\left(\frac{1}{\mathscr{M}\left(f\left(x_{n}\right), f\left(x_{n}\right), f\left(x_{n+1}\right), t\right)}-1\right) \\
& \leq \phi\left(\frac{1}{\mathscr{M}\left(y_{n-1}, y_{n-1}, y_{n}, t\right)}-1\right) \\
&-\psi\left(\frac{1}{\mathscr{M}\left(y_{n-1}, y_{n-1}, y_{n}, t\right)}-1\right) \\
& \leq \phi\left(\frac{1}{\mathscr{M}\left(y_{n-1}, y_{n-1}, y_{n}, t\right)}-1\right) .
\end{aligned}
$$

which, considering that $\phi$ function is non - decreasing, implies that $\mathscr{M}\left(y_{n}, y_{n}, y_{n+1}, t\right)>\mathscr{M}\left(y_{n-1}, y_{n-1}, y_{n}, t\right)$ for $\left(2_{1}^{\text {all }} n \in \mathbb{N}\right.$ and hence $\mathscr{M}\left(y_{n-1}, y_{n-1}, y_{n}, t\right)$ is an increasing sequence of positive real numbers in $(0,1]$.

Let $U(t)=\lim _{n \rightarrow \infty} \mathscr{M}\left(y_{n-1}, y_{n-1}, y_{n}, t\right)$, we show that $U(t)=$ 1 for all $t \gg \theta$. If not, there exist $t \gg \theta$ such that $U(t)<1$, then from the above inequality on taking $n \rightarrow \infty$. We obtain

$$
\phi\left(\frac{1}{U(t)}-1\right) \leq \phi\left(\frac{1}{U(t)}-1\right)-\psi\left(\frac{1}{U(t)}-1\right) .
$$

which is a contradiction. Therefore $\mathscr{M}\left(y_{n}, y_{n}, y_{n+1}, t\right) \rightarrow 1$ as $n \rightarrow \infty$. Now, for each positive integer p, by definition (2.13), we have

$$
\begin{array}{r}
\mathscr{M}\left(y_{n}, y_{n}, y_{n+p}, t\right) \geq \mathscr{M}\left(y_{n}, y_{n}, y_{n+1}, \frac{t}{p}\right) * \mathscr{M}\left(y_{n+1}, y_{n+1}, y_{n+2}, \frac{t}{p}\right) \\
* \cdots * \mathscr{M}\left(y_{n+p-1}, y_{n+p-1}, y_{n+p}, \frac{t}{p}\right) .
\end{array}
$$

It follows that

$\lim _{n \rightarrow \infty} \mathscr{M}\left(y_{n}, y_{n}, y_{n+p}, t\right) \geq 1 * 1 * \cdots * 1=1$. At the same 
time, we have

$$
\begin{array}{r}
\phi\left(\mathscr{N}\left(y_{n}, y_{n}, y_{n+1}, t\right)=\phi\left(\mathscr{N}\left(g\left(x_{n}\right), g\left(x_{n}\right), g\left(x_{n+1}\right), t\right)\right)\right. \\
\leq \phi\left(\mathscr{N}\left(f\left(x_{n}\right), f\left(x_{n}\right), f\left(x_{n+1}\right), t\right)\right) \\
-\psi\left(\mathscr{N}\left(f\left(x_{n}\right), f\left(x_{n}\right), f\left(x_{n+1}\right), t\right)\right) \\
\leq \phi\left(\mathscr{N}\left(y_{n-1}, y_{n-1}, y_{n}, t\right)\right. \\
-\psi\left(\mathscr{N}\left(y_{n-1}, y_{n-1}, y_{n}, t\right)\right) \\
<\phi\left(\mathscr{N}\left(y_{n-1}, y_{n-1}, y_{n}, t\right)\right.
\end{array}
$$

which, considering that the $\phi$ function is non-decreasing, implies that $\mathscr{N}\left(y_{n}, y_{n}, y_{n+1}, t\right)<\mathscr{N}\left(y_{n-1}, y_{n-1}, y_{n}, t\right)$ for all $n \in \mathbb{N}$ and hence $\mathscr{N}\left(y_{n-1}, y_{n-1}, y_{n}, t\right)$ is a decreasing sequence of positive real numbers in $[0,1)$.

Let $V(t)=\lim _{n \rightarrow \infty} \mathscr{N}\left(y_{n-1}, y_{n-1}, y_{n}, t\right)$, we show that $V(t)=$ 0 for all $t \gg \theta$. If not, there exists $t \gg \theta$ such that $V(t)>0$, then from the above inequality on taking $n \rightarrow \infty$, we obtain $\phi(V(t)) \leq \phi(V(t))-\psi(V(t))$. which is a contraction.

Therefore $\mathscr{N}\left(y_{n}, y_{n}, y_{n+1}, t\right) \rightarrow 0$ as $n \rightarrow \infty$. Now, for each positive integer $p$, by definition (9), we have

$$
\begin{array}{r}
\mathscr{M}\left(y_{n}, y_{n}, y_{n+p}, t\right)+\mathscr{N}\left(y_{n}, y_{n}, y_{n+p}, t\right) \leq 1 \\
\text { and } \lim _{n \rightarrow \infty}\left[\mathscr{M}\left(y_{n}, y_{n}, y_{n+p}, t\right)+\mathscr{N}\left(y_{n}, y_{n}, y_{n+p}, t\right)\right] \leq 1
\end{array}
$$

It follows that $\lim _{n \rightarrow \infty} \mathscr{N}\left(y_{n}, y_{n}, y_{n+p}, t\right)=0$. Hence $y_{n}$ is a Cauchy sequence. If $\mathrm{f}(\mathrm{X})$ is complete, then there exists $\mathrm{q} \in$ $\mathrm{f}(\mathrm{X})$ such that $y_{n} \rightarrow q$ as $n \rightarrow \infty$. The same holds if $\mathrm{g}(\mathrm{X})$ is complete with $\mathrm{q} \in \mathrm{g}(\mathrm{X})$. Let $\mathrm{p} \in \mathrm{X}$ be such that $f(p)=q$. Now, we shall show that $\mathrm{p}$ is a coincidence point of $\mathrm{f}$ and $\mathrm{g}$. In fact, we have $q$. If it is not so, then we consider

$$
\begin{aligned}
\phi\left(\frac{1}{\mathscr{M}(f(q), f(q), q, t)}-1\right) & =\phi\left(\frac{1}{\mathscr{M}(g(q), g(q), g(p), t)}-1\right) \\
& \leq \phi\left(\frac{1}{\mathscr{N}(f(q), f(q), f(p), t)}-1\right) \\
& -\psi\left(\frac{1}{\mathscr{M}(f(q), f(q), f(p), t)}-1\right) \\
& \leq \phi\left(\frac{1}{\mathscr{M}(f(q), f(q), q, t)}-1\right) \\
& -\psi\left(\frac{1}{\mathscr{M}(f(q), f(q), q, t)}-1\right)
\end{aligned}
$$

which is a contradiction that leads our result. The uniqueness of fixed point follows from the inequality (1) and (2) and so the proof is complete.

Example 3.2. $\operatorname{Let}(X, \mathscr{M}, \mathscr{N}, *, \diamond)$ be a complete intuitionistic generalized fuzzy cone metric space and let $X=\left\{\frac{1}{n} ; n \in\right.$ $\mathbb{N}\} \cup\{0\}, *$ be minimum norm and $\diamond$ be a maximum norm. Let $\mathscr{M}, \mathscr{N}$, be defined by

$$
\begin{aligned}
& \mathscr{M}(x, y, z, t)= \begin{cases}\frac{t}{t+(|x+y|+|y+z|+|z+x|)}, & \text { if } t>0 \\
0, & \text { if } t=0\end{cases} \\
& \mathscr{N}(x, y, z, t)= \begin{cases}\frac{|x+y|+|y+z|+|z+x|}{t+(|x+y|+|y+z|+|z+x|)}, & \text { if } t>0 \\
0, & \text { if } t=0\end{cases}
\end{aligned}
$$

Also, define $\phi, \psi:[0, \infty) \rightarrow[0, \infty)$ by $\phi(t)=\frac{t}{2}, \psi(t)=\frac{t}{8}$, for all $t \gg \theta, f(x)=\frac{x}{2}$ and $g(x)=\frac{x}{4}$. Obviously $g(X) \subseteq f(X)$ and $\psi$ is a continuous function. Then we have

$\phi\left(\frac{1}{\mathscr{M}(f(x), f(y), \mathscr{M} f(z), t)}-1\right)-\psi\left(\frac{1}{\mathscr{M}(f(x), f(y), f(z), t)}-1\right)$

$$
\begin{aligned}
& \phi\left(\frac{1}{\mathscr{M}\left(g(p), g(p), f\left(x_{n+1}\right), t\right)}-1\right)=\phi\left(\frac{1}{\mathscr{M}\left(g(p), g(p), g\left(x_{n}\right), t\right)}-1\right)=\frac{3(|x+y|+|y+z|+|z+x|)}{16 t} \\
& \leq \phi\left(\frac{1}{\mathscr{M}\left(f(p), f(p), f\left(x_{n}\right), t\right)}-1\right) \geq \frac{2(|x+y|+|y+z|+|z+x|)}{16 t} \\
& -\psi\left(\frac{1}{\mathscr{M}\left(f(p), f(p), f\left(x_{n}\right), t\right)}-1\right)=\phi\left(\frac{1}{\mathscr{M}(g(x), g(y), g(z), t)}-1\right)
\end{aligned}
$$

for every $\mathrm{t} \gg 0$, which on taking $n \rightarrow \infty$ gives that,

From the above inequality and the fact that $\mathscr{N}=1-\mathscr{M}$ we conclude that the conditions (1) and (2) are satisfied. Thus $g$ is intuitionistic generalized $(\phi-\psi)$-weak contraction with

$$
\begin{array}{rlrl}
\lim _{n \rightarrow \infty} \mathscr{M}\left(g(p), g(p), f\left(x_{n+1}\right), t\right) & =\lim _{n \rightarrow \infty} \mathscr{M}\left(g(p), g(p), g\left(x_{n}\right), t\right)^{\text {respect to } f .} \\
& =\mathscr{M}(g(p), g(p), f(p), t) & \text { Corollary } 3.3 \\
& =1 . & & \text { eralized fuzzy }
\end{array}
$$

Therefore $f(p)=g(p)=q$. Now, we shall show that $f(q)=$

Corollary 3.3. Let $(X, \mathscr{M}, \mathscr{N}, *, \diamond)$ be an intuitionistic generalized fuzzy cone metric space and $g: X \rightarrow X$ be a intuitionistic $(\phi, \psi)$-weak contraction. If $\psi$ is continuous then $g$ has a unique fixed point.

Corollary 3.4. $\operatorname{Let}(X, \mathscr{M}, \mathscr{N}, *, \diamond)$ be an intuitionistic generalized fuzzy cone metric space and $g: X \rightarrow X$ be a mapping 
satisfying

$$
\begin{aligned}
\phi\left(\frac{1}{\mathscr{N}(g(x), g(y), g(z), t)}-1\right) & \leq k \phi\left(\frac{1}{\mathscr{M}(x, y, z, t)}-1\right) \text { and } \\
\phi(\mathscr{N}(g(x), g(y), g(z), t)) & \leq k \phi(\mathscr{N}(x, y, z, t))
\end{aligned}
$$

for each $x, y, z \in X, t \in \theta$ and $k \in(0,1)$.

Theorem 3.5. Let $(X, \mathscr{M}, \mathscr{N}, *, \diamond)$ be an intuitionistic generalized fuzzy cone metric space and $f_{i}, g_{j}$ be two finite families of self mappings on $X$ with $f=f_{1} . f_{2} \cdots f_{n}$ and $g=g_{1} \cdot g_{2} \ldots g_{m}$, where $i \in\{1,2, \ldots, m\}$ and $j \in\{1,2, \ldots, n\}$. Let $g$ be a generalized intuitionistic $(\phi, \psi)$ - weak contraction with respect to $f$. If $g(X) \subseteq f(X)$ and $f(X)$ or $g(X)$ is a complete subset of $X$ then $g_{j}$ and $f_{i}$ have a unique common fixed point in $X$, provided that $\psi$ is a continuous function and the families $f_{i}$, and $g_{j}$ commute pairwise.

Proof. Using Theorem (16), we conclude that $f$ and $g$ have a unique common fixed point, say $q$. Now, we want to show that $q$ remains the fixed point of all the component mappings. For this consider,

$$
\begin{aligned}
g g_{i}(q) & =\left(g_{1} g_{2} \cdots g_{m}\right) g_{i}(q) \\
& =\left(g_{1} g_{2} \cdots g_{m-1}\right) g_{m} g_{i}(q) \\
& =\left(g_{1} g_{2} \cdots g_{m-1}\right) g_{i} g_{m}(q) \\
& =\cdots \\
& =g_{1} g_{i}\left(g_{2} g_{3} \cdots g_{m}\right)(q) \\
& =g_{i} g_{1}\left(g_{2} g_{3} \cdots g_{m}\right)(q) \\
& =g_{i} g(q) \\
& =g_{i}(q) .
\end{aligned}
$$

Similarly, we can show that $g f_{j}(q)=f_{j} g(q)=f_{j}(q), f f_{j}(q)=$ $f_{j} f(q)=f_{j}(q)$ and $f g_{i}(q)=g_{i} f(q)=g_{i}(q)$, which implies that, for all $\mathrm{i}$ and $\mathrm{j}, g_{i}(q)$ and $I_{j}(q)$ are other fixed points of the pair $\{g, f\}$. Now the uniqueness of common fixed point of mappings $\mathrm{f}$ and $\mathrm{g}$, we get $q=g_{i}(q)=I_{j}(q)$, which shows that $q$ is a common fixed point of $f_{i}$ and $g_{j}$ for all $\mathrm{i}$ and $\mathrm{j}$.

Example 3.6. Set $\mathscr{M}, \mathscr{N}, *, \diamond$ as in Example (17) and let $X=[0, \infty)$ and

$P=\mathbb{R}^{+}$. Define $\psi:[0, \infty) \rightarrow[0, \infty)$ by $\psi(t)=\frac{t}{4}$, for all $t \gg \theta$ and two families of self mappings $f_{i}$ and $g_{j}$, where $i, j \in$ $\{1,2, \ldots, m\}$ by

$f_{i}(x)=\left\{\begin{array}{ll}0, & \text { if } x=0 \\ \frac{1}{x \sqrt[n]{6}}, & \text { if } x \in \text { intP }\end{array} \quad\right.$ and $\quad g_{j}(x)= \begin{cases}0, & \text { if } x=0 \\ \frac{1}{x \sqrt[n]{2}}, & \text { if } x \in \text { int } P\end{cases}$

Then, we have

$$
\phi\left(\frac{1}{\mathscr{M}(f(x), f(y), f(z), t)}-1\right)-\psi\left(\frac{1}{\mathscr{M}(f(x), f(y), f(z), t)}^{[12]}-1\right)_{\mathrm{ji}}^{\mathrm{N}}
$$

$$
\begin{aligned}
& =\frac{3\left(z^{6}\left|x^{6}+y^{6}\right|+x^{6}\left|y^{6}+z^{6}\right|+y^{6}\left|z^{6}+x^{6}\right|\right)}{2 t x^{6} y^{6} z^{6}} \\
& \geq \frac{\left(z^{2}\left|x^{2}+y^{2}\right|+x^{2}\left|y^{2}+z^{2}\right|+y^{2}\left|z^{2}+x^{2}\right|\right)}{2 t x^{2} y^{2} z^{2}} \\
& =\phi\left(\frac{1}{\mathscr{M}(g(x), g(y), g(z), t)}-1\right)
\end{aligned}
$$

From the above inequality and fact that $\mathscr{N}=1-\mathscr{M}$, we conclude that the conditions (1) and (2) are satisfied. All the hypothesis of Theorem (20) are satisfied, then $f_{i}$ and $g_{j}$ have a unique common fixed point.

\section{Conclusion}

In this paper, an approach has been developed to extend and generalized the $(\phi, \psi)$-weak contraction in intuitionistic generalized fuzzy cone metric space and prove some common fixed point results. The results are illustrated with a wellanalyzed example in Section 3.

\section{References}

[1] A.M. Ali and G.R. Kanna, Intuitionistic Fuzzy Cone Metric Spaces and Fixed Point Theorems, International Journal of Mathematics And its Applications 55,(2017),25-36.

[2] Beg. I., Vetro. C., Gopal. D., and Imdad. M. $(\phi, \psi)$-weak contractions in intuitionistic fuzzy metric spaces. Journal Of Intelligent and Fuzzy Systems, 26(5)(2014), $2497-$ 2504.

[3] K. Atanassov, Intuitionistic fuzzy sets, Fuzzy, Sets and Systems, 20, (1986), 87-96.

[4] A. George, and P. Veeramani, On some results in fuzzy metric spaces, Fuzzy sets and Systems, 1994, 395-399.

[5] L. Huang, X. Zhang, Cone metric spaces and fixed point theorems of contractive mappings, J. Math. Anal. Appl., 332 (2007) 1468-1476.

[6] M. Imdad and J. A. Ali, General fixed point theorem in fuzzy metric space via an implicit function, Journal of Applied Mathematics and informatics, 26(2008), 591603.

[7] I. Kramosil, J. Michlek, Fuzzy metric and statistical metric spaces, Kybernetika, 11(1975) 336-344.

[8] M.S. Khan, M. Swaleh and S. Sessa, Fixed point theorems by altering distances between the points, Bulletin of the Australian Mathematical Society, 30(1984),1-9.

[9] T. Oner, M.B. Kandemir, B. Tanay, Fuzzy cone metric spaces, J. Nonlinear Sci. and Appl. 8(2015),610-616.

[10] T. Oner, On some results in fuzzy cone metric spaces, Int. J. Adv. Comput. Eng. Network, 4 (2016),37-39.

[11] J. H. Park, Intuitionistic fuzzy metric spaces, Chaos Solitons Fractals, 22(2004), 1039-1046.

12] N. Priyobarta, Yumnam Rohen and Thokchom Chhatrajit, Common Fixed Point Theorems for $(\phi, \psi)$ - Weak

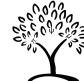


Contractions in Intuitionistic Fuzzy Cone Metric Spaces, International Journal of Advances in Mathematics, 1 (2018), 73-82.

[13] S. Rezapour, S., R. Hamlbarani, Some notes on paper Cone metric spaces and fixed point theorems of contractive mappings, J. Math. Anal. Appl. 345(2008) 719-724.

[14] Shaban Sedghi, Nabi Shobe, and Haiyun Zhou, A Common Fixed Point Theorem in $D^{*}$ Metric Spaces, FixedPoint Theory Appl., , 2007,Article ID 27906, 13 pages.

[15] C. Vetro, D. Gopal, and M. Imdad, Common fixed point theorems for $(\phi, \psi)$-weak contractions in fuzzy metric spaces, Indian Journal of Mathematics, 52(2010), 573590.

[16] Zadeh, L. A, Fuzzy sets, Information and Computation, 8(1965) 338-353.

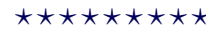

$\operatorname{ISSN}(\mathrm{P}): 2319-3786$

Malaya Journal of Matematik

$\operatorname{ISSN}(\mathrm{O}): 2321-5666$

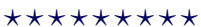

